\section{Video capsule endoscopy findings in Ehlers-Danlos syndrome with recurrent gastrointestinal bleeding}

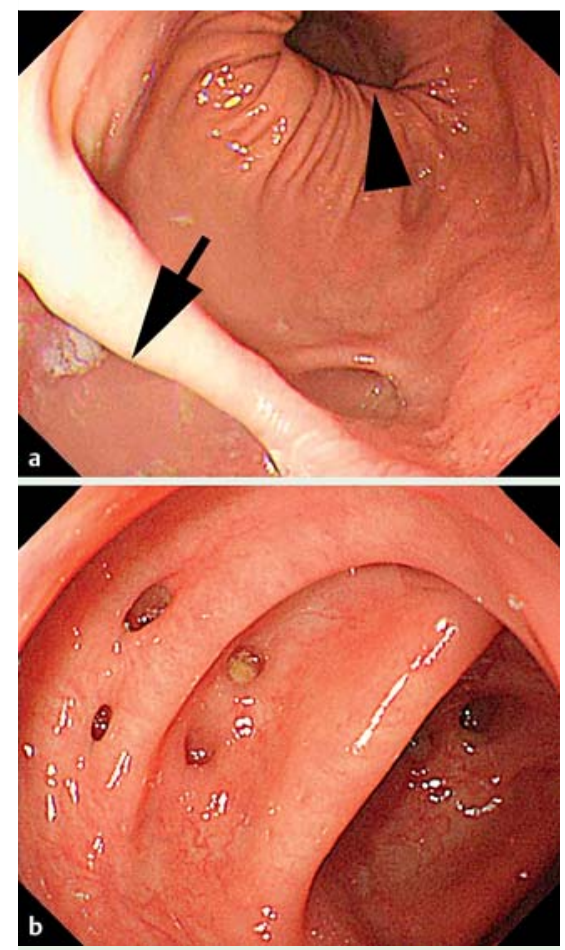

Fig. 1 Endoscopic findings in a middle-aged man with Ehlers-Danlos syndrome complaining of melena and dizziness. a Upper gastrointestinal endoscopy showing a subphrenic esophageal diverticulum (arrow), one of the common findings in Ehlers-Danlos syndrome, adjacent to the esophagogastric junction (arrowhead). b Colonoscopy showing a diverticulum of the sigmoid colon.

A man in his forties with Ehlers-Danlos syndrome (classic type) was admitted to our hospital because of melena and dizziness. His past history included two episodes of gastrointestinal bleeding of unknown origin. Upper and lower gastrointestinal endoscopy revealed a diverticulum of the esophagus and multiple diverticula in the sigmoid colon ( $\boldsymbol{\nabla}$ Fig. $\mathbf{1}$ ) but no evidence of recent bleeding. Colonoscopy also revealed several erosions in the terminal ileum ( $\bullet$ Fig. 2 ). We therefore carried out video capsule endoscopy, which revealed multiple diverticula with adjacent erosions in the distal jejunum ( $\bullet$ Video 1 ), and numerous erosions in most of the ileum ( Fig.3). On the basis of these findings, the most likely origin of the bleeding was the small intestine.

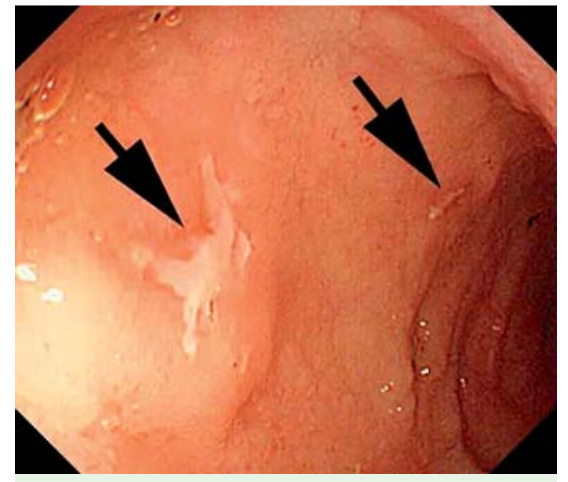

Fig. 2 Colonoscopy of the terminal ileum showing scattered erosions with slight redness (arrows).

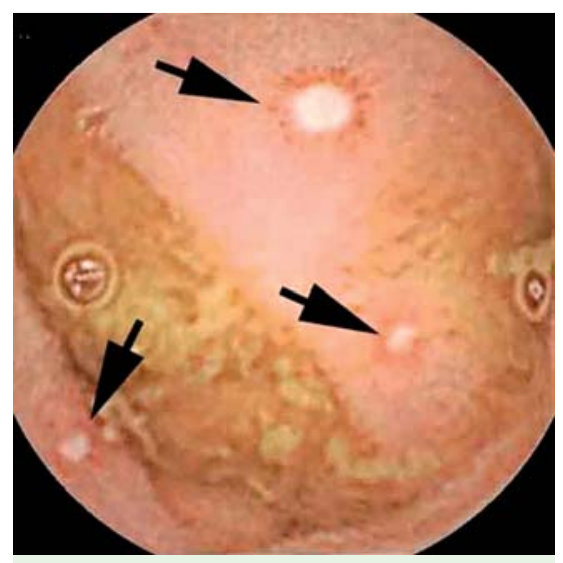

Fig. 3 Capsule endoscopy showing multiple erosions in the ileum (arrows).

Ehlers-Danlos syndrome is a rare inherited connective tissue disorder with hypermobile joints, hyperextensive skin, and fragile tissues; diverticulum formation in the gastrointestinal tract due to the fragility of the connective tissues has been reported $[1,2]$. One of the uncommon but potentially fatal complications of Ehlers-Danlos syndrome is gastrointestinal bleeding [3], but the focus of the bleeding has not yet been elucidated. The

\section{Video 1}

Capsule endoscopy showing a diverticulum The diverticula were seen mainly in the lower jejunum. with adjacent erosions in the lower jejunum. present case suggests that the small intestine is an important candidate bleeding site in Ehlers-Danlos syndrome. Some patients with Ehlers-Danlos syndrome (vascular type) require close medical follow-up to prevent sudden death by organ rupture at a young age. Thus, we believe that Ehlers-Danlos syndrome should be considered in the differential diagnosis when a clinician encounters the unusual capsule endoscopic finding of multiple diverticula with erosions in the small intestine. The findings in our present patient also suggest that the focus of smallintestinal bleeding in Ehlers-Danlos syndrome is not the diverticula but the multiple erosions in the small intestine.

\section{Endoscopy_UCTN_Code_CCL_1AC_2AH}

\section{Competing interests: None}

H. Kishikawa', J. Nishida', S. Takarabe', K. Arahata ${ }^{1}$, A. Ito ${ }^{1}$, J. Miyoshi ${ }^{1}$,

\section{S. Kaida ${ }^{1}$, T. Hibi ${ }^{2}$}

${ }^{1}$ Department of Gastroenterology, Tokyo Dental College, Ichikawa General Hospital, Chiba, Japan

${ }^{2}$ Department of Internal Medicine, School of Medicine, Keio University, Tokyo, Japan

\section{References}

1 Parapia LA, Jackson C. Ehlers-Danlos syndrome - a historical review. Br J Haematol 2008; 141: $32-35$

2 Pepin M, Schwarze U, Superti-Furga A et al. Clinical and genetic features of Ehlers-Danlos syndrome type IV, the vascular type. N Engl J Med 2000; 342: 673-680

3 Solomon JA, Abrams L, Lichtenstein GR. GI manifestations of Ehlers-Danlos syndrome. Am J Gastroenterol 1996; 91: 2282-2288

\section{Bibliography}

Dol http://dx.doi.org/

10.1055/s-0032-1325774

Endoscopy 2012; 44: E416

(c) Georg Thieme Verlag KG

Stuttgart - New York

ISSN 0013-726X

\section{Corresponding author}

\section{H. Kishikawa}

Department of Gastroenterology

Ichikawa General Hospital

Tokyo Dental College

5-11-13 Sugano Ichikawa

Chiba 272-8513

Japan

Fax: +81-47-3254456

kisikawa@tdc.ac.jp 